Research Paper

\title{
Long-Term Outcome of Oxaliplatin and Capecitabine (XELOX) Concomitant with Neoadjuvant Radiotherapy and Extended to the Resting Period in High Risk Locally Advanced Rectal Cancer
}

\author{
Jinghua Tang $12^{*}$, Xiaojun Wu 1,2*, Yanfang Bai ${ }^{1,3}$, Yuanhong Gao ${ }^{1,4}$, Wu Jiang 1,2, Lingheng Kong 1,2, \\ Junzhong Lin 1,2, Desen Wan 1,2, Zhizhong Pan ${ }^{1,2}{ }^{凶}$, Peirong Ding ${ }^{1,2} \bowtie$ \\ 1. Sun Yat-sen University Cancer Center, State Key Laboratory of Oncology in South China, Collaborative Innovation Center of Cancer Medicine, Guangzhou, \\ P R. China; \\ 2. Department of Colorectal Surgery, Sun Yat-sen University Cancer Center, Guangzhou, P. R. China \\ 3. Department of Anesthesiology \& Operating Theatre, Sun Yat-sen University Cancer Center, Guangzhou, P. R. China \\ 4. Department of Radiation Oncology, Sun Yat-sen University Cancer Center, Guangzhou, P. R. China \\ *Jinghua Tang and Xiaojun Wu contributed equally to this article.
}

$\triangle$ Corresponding author: Zhizhong Pan and Peirong Ding. Address: Departments of Colorectal Surgery, Sun Yat-sen University Cancer Center, No. 651 Dongfeng Road East, Guangzhou, 510060, Guangdong, P. R. China. Tel: 86-20-87343124; Fax: 86-20-87343372; E-mail: panzz@susycc.org.cn; dingpr@sysucc.org.cn

( $)$ Ivyspring International Publisher. This is an open access article distributed under the terms of the Creative Commons Attribution (CC BY-NC) license (https://creativecommons.org/licenses/by-nc/4.0/). See http://ivyspring.com/terms for full terms and conditions.

Received: 2017.11.15; Accepted: 2018.01.19; Published: 2018.04.06

\begin{abstract}
Purpose: This study aimed at investigating the long-term outcomes of oxaliplatin and capecitabine (XELOX) administered concurrently with preoperative radiation and extended to the resting period in patients with high-risk locally advanced rectal cancer (LARC).

Methods: From January 2010 to December 2013, 45 patients were recruited. Study treatment consisted two cycles of XELOX regimen concomitant with preoperative radiation and then followed by an additional cycle of XELOX regimen between completion of neoadjuvant radiotherapy and surgery. Disease-free survival (DFS) time and overall survival (OS) time were analyzed.

Results: The median follow-up was 51 months. Twelve (26.7\%) patients developed local recurrence or distant metastasis, including $10(22.2 \%)$ patients developing distant metastasis only, 1 (2.2\%) patient local recurrence only, and 1 (2.2\%) patient both local recurrence and distant metastasis. The estimated 3-year DFS and OS was 75.5\% (95\% Cl, 63.0\%-88.0\%) and $88.6 \%(95 \% \mathrm{Cl}$, 98.0\%-79.2\%), respectively. Receiving adjuvant chemotherapy was a significant predictor for DFS, with hazard ratio 0.24 (95\% Cl: $0.08-0.74)$.

Conclusion: This intensified strategy with oxaliplatin and capecitabine (XELOX) administered concomitantly with neoadjuvant radiotherapy and then extended to the resting period in high-risk LARC patients is efficient. The long-term outcome is promising. Further study of this strategy is warranted.
\end{abstract}

Key words: Rectal Cancer; Chemoradiotherapy; Oxaliplatin; Capecitabine; Long-term Survival

\section{Introduction}

Substantial progress has been made in the management of locally advanced rectal cancer (LARC) during the past decades. With combined- modality therapy consisting of preoperative chemoradiotherapy (CRT), total mesorectal excision surgery (TME) and postoperative chemotherapy, local 
recurrence rate has been dramatically reduced [1-5]. However, few of the published randomized trials have demonstrated a survival benefit after a long term follow-up $[5,6]$. Despite the local recurrence rates having been reduced to less than $5 \%$, distant metastasis still occur in about $25 \%$ of patients. Given that better control of systemic disease becomes the major challenge for the management of LARC, there is a strong clinical rationale for upfront use of intensified systemic therapy to prevent or eradicate micrometastases. Adding oxaliplatin to the regimen of fluorouracil based adjuvant chemotherapy has improved disease free survival and overall survival in colon cancer [7-9]. Therefore, several large randomized phases III trials tested the efficacy of adding oxaliplatin to the multimodal neoadjuvant treatment for LARC. However, except the German $\mathrm{CAO} / \mathrm{ARO} / \mathrm{AIO}-04$ study showing an improved disease free survival, integrating oxaliplatin into the combined modality strategies failed to show a survival benefit [10-13]. As a result, the strategy of simply adding oxaliplatin concomitant to conventional CRT remains unsatisfactory.

Previously, to enhance systemic control, we used an intensified chemotherapy with oxaliplatin and capecitabine (XELOX) administered concomitantly to preoperative radiotherapy and extended to the resting period between complication of CRT and surgery for high-risk LARC patients. Preliminary results suggest this alternative strategy was well tolerated and associated with high rates of pathological complete response (pCR) and major regression rates [14]. In the present study, we aimed to report the long-term outcomes of the patients treated according to this protocol.

\section{Patients and methods}

\section{Patients}

This was a retrospective study. Data were retrieved from a prospectively maintained data base at Sun Yet-sen University cancer center. Details on patients, methods and treatment were published previously [14]. In brief, patients with high risk LARC and treated with concurrent CRT followed by chemotherapy in the resting period between the completion of radiotherapy and surgery were included in this study. The tumor considered high risk had to show at least one of the following factors: low-lying tumor (within $6 \mathrm{~cm}$ of anal verge), bulky tumor (larger than $5 \mathrm{~cm}$ ), infiltration of adjacent tissues or organs (cT4), positive lymph node, or elevated serum carcinoembryonic antigen. Patients with metastatic or recurrence rectal cancer and patients who had undergone transanal local excision or endoscopic mucosal resection were excluded from this study. Pretreatment distant metastasis assessment included chest and abdominal computer tomography scans (CT). Magnetic resonance imaging (MRI) with or without endoscopic ultrasound (EUS) was mandatory for local staging. The investigational protocol was approved by the Research and Ethics Committee of the Sun Yet-sen University cancer center. Written informed consent was obtained from all patients before the initiation of treatment according to the institutional guidelines.

\section{Treatment}

The preoperative radiotherapy consisted of 46 Gy in 23 fractions, using 8- and 15-MV X rays in a 3 -field irradiation technique, delivered to the primary tumor lesion and to mesorectal, the two end portions of rectum and the pelvis internal iliac lymph drainage areas. From 2012, a 4 Gy (2 Gy per fraction) boost to the gross tumor volume increased the total dose to 50 Gy. Two cycles of standard XELOX regimen (oxaliplatin $130 \mathrm{mg} / \mathrm{m}^{2}$ day 1 ; capecitabine 1000 $\mathrm{mg} / \mathrm{m}^{2}$ twice daily, days 1-14; repeat every 3 weeks) were administered concomitantly to radiotherapy prior to 2012. To avoid potential treatment interruption or delay, a modified XELOX regimen (oxaliplatin $100 \mathrm{mg} / \mathrm{m}^{2}$ day 1 ; capecitabine 1000 $\mathrm{mg} / \mathrm{m}^{2}$ twice daily, days 1-14; repeat every 3 weeks) was introduced to the concurrent chemotherapy from 2012 [15]. Consolidation chemotherapy with a standard XELOX regimen was given after one week completion of the CRT.

About 4-5 weeks after the completion of radiation, comprehensive local and distant reassessment was performed by CT scan of the chest, abdomen and MRI of the pelvis. Radical rectal resection according to the TME principle was scheduled 6-8 weeks after the end of radiation. Adjuvant chemotherapy was recommend for all patients with 5 cycles standard XELOX regimen or 3 cycles standard XELOX plus 2 cycles capecitabine administered at a dose of $1250 \mathrm{mg} / \mathrm{m}^{2}$ twice daily for 14 days every 3 weeks if unacceptable oxaliplatin-induced neurotoxicity occurred.

\section{Pathological assessment}

The $7^{\text {th }}$ edition of American Joint Committee on Cancer TNM system was used for tumor staging. Tumor regression after preoperative treatment was evaluated according to the Dworak regression grade system [16].

\section{Follow up}

Patients were followed up at interval of 3 months for two years after surgery, and then every 6 months for the next 3 years. Patient history, physical 
examination, abdominal ultrasonography and serum carcinoembryonic antigen was proposed at each visit. Colonoscopy and CT scan of chest, abdomen and pelvis were recommended annually for up 5 years after surgery.

\section{Statistical analysis}

Kaplan-Meier method was used to estimate the overall survival (OS) and disease-free survival (DFS). The log-rank test was used to compare the survival curves. Multivariate Cox proportional hazard regression was used to analyze the major factors affecting survival. Statistical Package for the Social Sciences (SPSS)' version 15.0 for Windows (SPSS, Inc. Chicago, IL) was used for all analysis. DFS was defined as the interval from the first day of the treatment to the date of relapse, death, or the last follow-up, whichever occurred first. OS was defined as the interval from the first day of the treatment to death from any cause. All data in our study have been uploaded onto the Research Data Deposit public platform at Sun Yat-sen University Cancer Center for future reference (number RDDA 2017000353).

\section{Results}

\section{Clinicopathologic demographic data}

From January 2010 to December 2013, a total of 45 eligible patients were analyzed. Numbers differ from the previous report because another 9 patients received the same treatment in the next year were included in the analysis. Patients and disease characteristics and pathological findings are listed in Table 1 which does not differ significantly from the previous report. Of note, all patients obtained R0 resection, including 1 patient with liver metastasis detected intraoperative. Primary tumor downstaging was observed in $32(71.7 \%)$ patients and nodal downstaging in $24(53.3 \%)$ patients. A total of $36(80 \%)$ patients received adjuvant chemotherapy and 34 (75.6\%) patients received greater than $80 \%$ of the initially planned dose.

\section{DFS and OS}

After a median of 51 months (IQR 45-57) follow-up, 12 (26.7\%) patients developed local recurrence or distant metastasis, including 10 (22.2\%) patients developing distant metastasis only, 1 (2.2\%) patient local recurrence only, and 1 (2.2\%) patient both local recurrence and distant metastasis. The median time to recurrence from surgery was 15.7 moths (range, 8.2-48.9 moths). Median DFS was not reached, and the estimated 3-years DFS was 75.5\% (95\% CI, 63.0\%-88.0\%) (Fig 1A).
Table 1. Disease characteristics and pathological findings.

\begin{tabular}{|c|c|}
\hline Characteristic & Value $(n=45)$ \\
\hline Age (years), media (range) & $58(27-76)$ \\
\hline \multicolumn{2}{|l|}{ Gender } \\
\hline Male & $32(71.1)$ \\
\hline Female & $13(28.9)$ \\
\hline \multicolumn{2}{|l|}{ Clinical TNM stage } \\
\hline II & $16(35.6)$ \\
\hline III & $29(64.4)$ \\
\hline \multicolumn{2}{|l|}{ CEA before treatment } \\
\hline$\leq 5$ & $31(68.9)$ \\
\hline$>5$ & $13(28.9)$ \\
\hline Missing & $1(2.2)$ \\
\hline \multicolumn{2}{|l|}{ CEA after CRT before surgery } \\
\hline$\leq 5$ & $40(88.9)$ \\
\hline$>5$ & $3(6.7)$ \\
\hline Missing & $2(4.4)$ \\
\hline Interval from RT to surgery (days), media (range) & $51(34-102)$ \\
\hline Number of lymph nodes retrieved, media (range) & $8(0-16)$ \\
\hline \multicolumn{2}{|l|}{ Pathological tumor response } \\
\hline ypCR & $17(37.8)$ \\
\hline TRG 3 & $17(37.8)$ \\
\hline TRG 1-2 & $11(24.4)$ \\
\hline \multicolumn{2}{|l|}{ Pathological TNM stage } \\
\hline ypCR & $17(37.8)$ \\
\hline I & $9(20.0)$ \\
\hline II & $11(24.4)$ \\
\hline III & $7(15.6)$ \\
\hline IV & $1(2.2)$ \\
\hline Adjuvant chemotherapy & $34(75.6)$ \\
\hline
\end{tabular}

At the time of analysis, $6(13.3 \%)$ patients died and all of them suffered recurrence. The median time to death from surgery was 30.4 moths (range, 17.0744.63 moths). The median OS was not reached also, and the estimated 3-years OS was $88.6 \%$ (95\% CI, 98.0\%-79.2\%) (Fig 1B).

Exploratory subgroup analysis according to pretreatment factors and pathological factors after preoperative chemoradiotherapy showed no significant predictors for DFS. Whether receiving adjuvant chemotherapy or not was the only significant predictor for DFS, with hazard ratio 0.24 (95\% CI: 0.08-0.74), and a positive trend was observed for primary tumor downstaging $0.50 \quad(95 \% \quad \mathrm{CI}$ : 0.19-1.58) (Table 2).

\section{Discussion}

The intensified strategy with XELOX regimen administered concurrently with preoperative radiation and extended to the resting period after CRT before surgery and as postoperative adjuvant chemotherapy resulted in excellent disease control and long-term survival, with well-tolerated toxicity and good compliance. After a median follow-up of 51 moths, the cumulative incidence of local recurrence and distant metastasis is $4.4 \%$ and $24.4 \%$, respectively. 
A

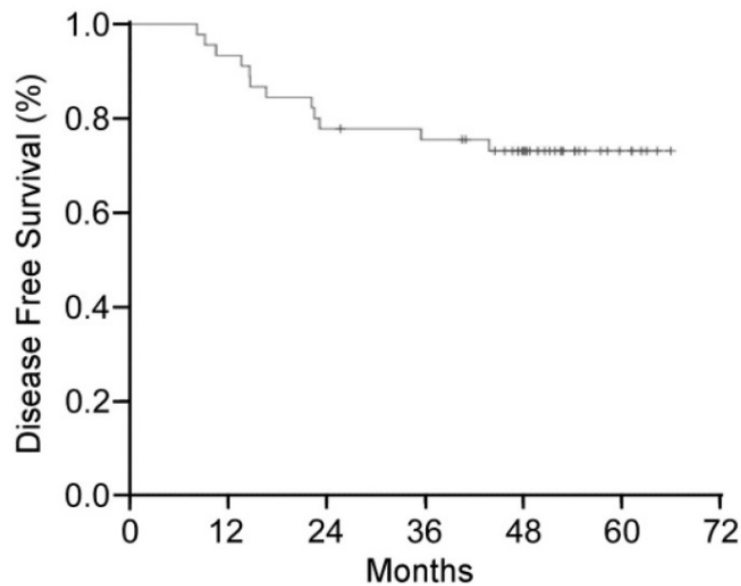

B

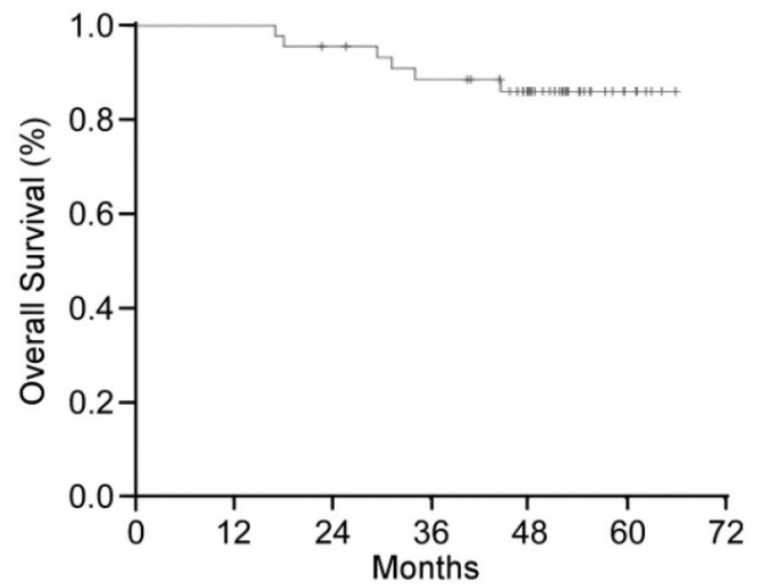

Figure 1. Kaplan-Meier Curve of (A) disease-free survival and (B) overall survival for 45 patients with high-risk locally advanced rectal cancer.

Table 2. Univariate and multivariate analysis of prognostic factors for DFS.

\begin{tabular}{|c|c|c|c|c|}
\hline \multirow[t]{2}{*}{ Prognostic factors } & \multicolumn{2}{|c|}{ Univariate analysis } & \multicolumn{2}{|c|}{ Multivariate analysis } \\
\hline & HR (95\% CI) & $P$ value & HR (95\% CI) & $P$ value \\
\hline Age (<60 vs. $\geq 60$ years) & $0.68(0.21-2.27)$ & 0.53 & & \\
\hline Gender (Male vs. female) & $0.59(0.19-1.85)$ & 0.36 & & \\
\hline $\begin{array}{l}\text { Interval time between CRT and } \\
\text { surgery }(\leq 6 w \text { vs. }>6 w)\end{array}$ & $2.64(0.72-9.76)$ & 0.15 & & \\
\hline $\begin{array}{l}\text { Tumor downstaging (yes vs. } \\
\text { no) }\end{array}$ & $0.50(0.19-1.58)$ & 0.24 & $0.33(0.10-1.15)$ & 0.08 \\
\hline $\begin{array}{l}\text { Nodal downstaging (yes vs. } \\
\text { no) }\end{array}$ & $0.58(0.18-1.83)$ & 0.35 & & \\
\hline $\begin{array}{l}\text { Pathological tumor response } \\
\text { (pCR or nearly-pCR vs. TRG } \\
1-2 \text { ) }\end{array}$ & $0.83(0.23-3.07)$ & 0.78 & & \\
\hline $\begin{array}{l}\text { ypTNM stage (ypCR vs. I vs. II } \\
\text { vs. III vs. IV) }\end{array}$ & $1.23(0.72-2.10)$ & 0.49 & & \\
\hline $\begin{array}{l}\text { Adjuvant chemotherapy (yes } \\
\text { vs. no) }\end{array}$ & $0.24(0.08-0.74)$ & 0.01 & $0.18(0.05-0.61)$ & $<0.01$ \\
\hline
\end{tabular}

With preoperative CRT/RT and optimized TME, local recurrence rates have been markedly reduced to below $5 \%$ in locally advanced rectal cancer $[2,4,5,17]$. In present study, the cumulative incidence of local recurrence was $4.4 \%$ which was comparable to the published studies. Hence, distant metastases are now the predominant causes for failure and better control of systemic disease becomes the major challenge for the management of LARC.

Several studies have investigated integrating combination chemotherapy into the combined modality aiming to improve local and systemic control. The strategy of adding oxaliplatin weekly to fluoropyrimidine-based chemotherapy was well studied. Except the CAO/ARO/AIO-04 trail, results from randomized phase III trials: STAR-01, ACCORD 12, NASPBR-04, and PETACC-6 showed that adding oxaliplatin to 5-FU/capecitabine-based preoperative CRT increased acute toxicity, but failed to improve the PCR rate and disease free or overall survival [10-13,
17]. The $\mathrm{CAO} / \mathrm{ARO} / \mathrm{AIO}-04$ trail with more than 1250 patients recruited reported a significant improvement of $\mathrm{pCR}$ rate $(13 \%$ vs. $17 \%, \mathrm{P}=0.04)$ and 3 -years DFS $(71.2 \%$ vs. $75.9 \%, \mathrm{P}=0.03)$. The current study reported a feasible $\mathrm{pCR}$ rate $(37.8 \%)$ and 3-years DFS $75.5 \% \quad(95 \% \quad C I, 63.0 \%-88.0 \%)$ which was comparable to that of the $\mathrm{CAO} / \mathrm{ARO} / \mathrm{AIO}-04$ trial. Of note, except the CAO/ARO/AIO-04 trial, most of the above mentioned studies did not change the schedule of fluoropyrimidine delivery with oxaliplatin weekly added to the conventional CRT, which might increase toxicities and result in more dose reduction or treatment interruptions. In the STAR-01, only $66 \%$ patients received initially planned oxaliplatin infusions with or without radiation dose reduction [10]. In the ACCORD-12, only $41 \%$ patients received full dose of oxaliplatin [11]. The current study using the XELOX regimen as the concurrent chemotherapy scheduled 1 week free of chemotherapy in the middle of radiation, which was similar to the CAO/ARO/AIO-04 trail. The alternative schedule was well tolerated and $85 \%$ patients received full dose of neoadjuvant oxaliplatin. In our study, all patients received the planned full dose of radiation and concurrent chemotherapy. Less dose reduction and treatment interruption might account for favorable outcome.

Lengthening the interval between CRT and surgery was associated with improved tumor response [18]. With the longer chemotherapy-free interval, there are also concerns about the possibility of increasing the risk of metastasis. Several small trails have tested the utility of consolidation chemotherapy into the resting period between the CRT and the surgery. Habr-Gama et al. first reported an impressive sustained complete response (65\%), with 3 additional cycles of fluoropyrimidine-based chemotherapy during the resting period [19]. Garcia-Aguilar et al. 
reported a prospective phase 2 trial consisting of 4 sequential study groups [20]. All patients received fluoropyrimidine-based chemoradiation. Study group 1 had surgery 6-8 weeks after CRT. Study group 2-4 received two, four, six cycles of mFOLFOX6, respectively, during the waiting period before surgery. The pCR rate was $18 \%, 25 \%, 30 \%$, and $38 \%$ in study group 1-4, respectively. Although promising, long-term follow up to measure the oncological outcome remains pending. Soo Jung Lee et al. recently reported a pilot study adding one cycle of fluoropyrimidine-based chemotherapy during the resting period between CRT and surgery. Despite more N2 disease and higher carcinoembryonic antigen level in the historical control group, the estimated 3 years DFS in the study was better than the control group (79.5\% vs. $67.5 \%, \mathrm{P}=0.04)$ [21].

Adjuvant chemotherapy after preoperative chemoradiotherapy and surgery for patients with rectal cancer was still controversial [22-25]. Although with insufficient strong evidence, current practices recommend a full course of adjuvant chemotherapy for all patients with rectal cancer receiving CRT and surgery, irrespective of the final pathology. The ADORE trail indicated that adjuvant FOLFOX improves DFS compared with fluorouracil plus leucovorin in patients with postoperative pathological stage II or III rectal cancer after preoperative chemoradiotherapy $(71.6 \%$ vs. $62.9 \%, \mathrm{P}=0.047)$ ) [26]. In the present study, a benefit of adjuvant chemotherapy with XELOX regimen was observed in exploratory subgroup analysis (HR 0.24, 95\% CI $0.08-0.74 ; \mathrm{P}=0.01$ ). Since these subgroup analyses are exploratory, it is hard to draw any conclusion.

The limitations of the present study are obvious. The inherent retrospective nature, relatively small numbers of patients, and single-arm design might limit the power to interpret data. Because of the absence of a control group, our results are merely hypothesis rather than confirmatory.

\section{Conclusion}

The intensified strategy with a XELOX regimen administered concurrently with radiation and extended to the resting period after CRT before surgery and as postoperative adjuvant chemotherapy was well tolerated and resulted in excellent long-term survival. These promising results warrant further investigation in future prospective randomized trials.

\section{Abbreviations}

LARC: locally advanced rectal cancer; DFS: disease-free survival; OS: overall survival; CRT: chemoradiotherapy; TME: total mesorectal excision; pCR: pathological complete response.

\section{Competing Interests}

The authors have declared that no competing interest exists.

\section{References}

1. Cedermark B, Dahlberg M, Glimelius B, et al. Improved survival with preoperative radiotherapy in resectable rectal cancer. N Engl J Med 1997; 336: 980-987.

2. Sauer R, Becker H, Hohenberger $W$, et al. Preoperative versus postoperative chemoradiotherapy for rectal cancer. N Engl J Med 2004; 351: 1731-1740.

3. Bosset J, Collette L, Calais G, et al. Chemotherapy with preoperative radiotherapy in rectal cancer. New Engl J Med 2006; 355: 1114-1123.

4. Gerard JP, Conroy T, Bonnetain F, et al. Preoperative radiotherapy with or without concurrent fluorouracil and leucovorin in T3-4 rectal cancers: results of FFCD 9203. J Clin Oncol 2006; 24: 4620-4625.

5. van Gijn W, Marijnen CA, Nagtegaal ID, et al. Preoperative radiotherapy combined with total mesorectal excision for resectable rectal cancer: 12-year follow-up of the multicentre, randomised controlled TME trial. Lancet Oncol 2011; 12: 575-582.

6. Peeters KC, Marijnen CA, Nagtegaal ID, et al. The TME trial after a median follow-up of 6 years: increased local control but no survival benefit in irradiated patients with resectable rectal carcinoma. Ann Surg 2007; 246: 693-701

7. Andre T, Boni C, Mounedji-Boudiaf L, et al. Oxaliplatin, fluorouracil, and leucovorin as adjuvant treatment for colon cancer. N Engl J Med 2004; 350: 2343-2351.

8. Andre T, Boni C, Navarro M, et al. Improved overall survival with oxaliplatin, fluorouracil, and leucovorin as adjuvant treatment in stage II or III colon cancer in the MOSAIC trial. J Clin Oncol 2009; 27: 3109-3116.

9. Yothers G, O'Connell MJ, Allegra CJ, et al. Oxaliplatin as adjuvant therapy for colon cancer: updated results of NSABP C-07 trial, including survival and subset analyses. J Clin Oncol 2011; 29: 3768-3774.

10. Aschele C, Cionini L, Lonardi S, et al. Primary tumor response to preoperative chemoradiation with or without oxaliplatin in locally advanced rectal cancer: pathologic results of the STAR-01 randomized phase III trial. J Clin Oncol 2011; 29: 2773-2780.

11. Gerard JP, Azria D, Gourgou-Bourgade S, et al. Clinical outcome of the ACCORD 12/0405 PRODIGE 2 randomized trial in rectal cancer. J Clin Oncol 2012; 30: 4558-4565.

12. Allegra CJ, Yothers G, O'Connell MJ, et al. Neoadjuvant 5-FU or Capecitabine Plus Radiation With or Without Oxaliplatin in Rectal Cancer Patients: A Phase III Randomized Clinical Trial. J Natl Cancer Inst 2015; 107.

13. Schmoll HJ, Stein A, Hofheinz RD, et al. Preoperative chemoradiotherapy and postoperative chemotherapy with capecitabine and oxaliplatin vs. capecitabine alone in locally advanced rectal cancer final analyses. Ann Oncol. 2016. 27: 467PD.

14. Gao YH, Zhang X, An X, et al. Oxaliplatin and capecitabine concomitant with neoadjuvant radiotherapy and extended to the resting period in high risk locally advanced rectal cancer. Strahlenther Onkol 2014; 190: 158-164.

15. Rodel C, Liersch T, Becker H, et al. Preoperative chemoradiotherapy and postoperative chemotherapy with fluorouracil and oxaliplatin versus fluorouracil alone in locally advanced rectal cancer: initial results of the German CAO/ARO/AIO-04 randomised phase 3 trial. Lancet Oncol 2012; 13 : 679-687.

16. Dworak O, Keilholz L, Hoffmann A. Pathological features of rectal cancer after preoperative radiochemotherapy. Int J Colorectal Dis 1997; 12: 19-23.

17. Rodel C, Graeven U, Fietkau R, et al. Oxaliplatin added to fluorouracil-based preoperative chemoradiotherapy and postoperative chemotherapy of locally advanced rectal cancer (the German CAO/ARO/AIO-04 study): final results of the multicentre, open-label, randomised, phase 3 trial. Lancet Oncol 2015; 16: 979-989.

18. Tulchinsky H, Shmueli E, Figer A, et al. An interval $>7$ weeks between neoadjuvant therapy and surgery improves pathologic complete response and disease-free survival in patients with locally advanced rectal cancer. Ann Surg Oncol 2008; 15: 2661-2667.

19. Habr-Gama A, Perez RO, Sabbaga J, et al. Increasing the rates of complete response to neoadjuvant chemoradiotherapy for distal rectal cancer: results of a prospective study using additional chemotherapy during the resting period. Dis Colon Rectum 2009; 52: 1927-1934.

20. Garcia-Aguilar J, Chow OS, Smith DD, et al. Effect of adding mFOLFOX6 after neoadjuvant chemoradiation in locally advanced rectal cancer: a multicentre, phase 2 trial. Lancet Oncol 2015; 16: 957-966.

21. Lee SJ, Kang BW, Chae YS, et al. Pilot Study of Neoadjuvant Chemoradiotherapy with Three Cycles of 5-Fluorouracil Plus Leucovorin for Treatment of Locally Advanced Rectal Cancer. Ann Surg Oncol 2016; 23 : 894-899.

22. Bosset JF, Calais G, Mineur L, et al. Fluorouracil-based adjuvant chemotherapy after preoperative chemoradiotherapy in rectal cancer: long-term results of the EORTC 22921 randomised study. Lancet Oncol 2014; 15: 184-190.

23. Breugom AJ, Swets M, Bosset JF, et al. Adjuvant chemotherapy after preoperative (chemo)radiotherapy and surgery for patients with rectal cancer: 
a systematic review and meta-analysis of individual patient data. Lancet Oncol 2015; 16: 200-207.

24. Breugom AJ, van Gijn W, Muller EW, et al. Adjuvant chemotherapy for rectal cancer patients treated with preoperative (chemo)radiotherapy and total mesorectal excision: a Dutch Colorectal Cancer Group (DCCG) randomized phase III trial. Ann Oncol 2015; 26: 696-701.

25. Bujko K, Glimelius B, Valentini V, et al. Postoperative chemotherapy in patients with rectal cancer receiving preoperative radio(chemo)therapy: A meta-analysis of randomized trials comparing surgery $+/$ - a fluoropyrimidine and surgery + a fluoropyrimidine + /- oxaliplatin. Eur J Surg Oncol 2015; 41: $713-723$.

26. Hong YS, Nam BH, Kim KP, et al. Oxaliplatin, fluorouracil, and leucovorin versus fluorouracil and leucovorin as adjuvant chemotherapy for locally advanced rectal cancer after preoperative chemoradiotherapy (ADORE): an open-label, multicentre, phase 2, randomised controlled trial. Lancet Oncol 2014; 15: 1245-1253. 